\title{
Breakthrough non-surgical laser sleep applications in dentistry and medicine
}

\author{
Harvey S Shiffman* \\ Boynton Laser Dental Center, Nova South-eastern College of Dental Medicine, Florida, USA
}

\begin{abstract}
There are a multitude of treatment options for sleep-disordered breathing (SDB), which can be classified into conservative, apparatus, and surgical methods. The choice of the method used will depend on the patient's tolerance of the procedure, and most importantly, on the severity of the patient's SDB. Nightlase C3 (concentric) falls into the Laser-assisted uvulopalatoplasty (LAUP) group but is unique as we are not removing any tissue but addressing several possible areas of collapse. However, we are modifying the tissue, the protocol addresses the floor of the mouth, posterior tongue and soft palate, uvula, and the tonsillar pillars. The LAUP method, which was originally introduced as a variant of the standard and relatively invasive surgical uvulopalatopharyngoplasty (UPPP) method, was modified in the early 90's to address mainly palatal flutter, which was found to be the most important factor in the mechanism of snoring. Therefore, instead of surgically shortening the palate as in UPPP, which inevitably risks impairing its function, the Nightlase C3 approach focuses on shrinking of the palatal tissue, reducing inflammation, reducing collapsibility and tongue repositioning. This is accomplished by thermally injuring the surface of the tissues, which heals by collagen shrinkage and neocollagenesis, thus producing the desired results. When performed at sub-ablative laser fluences, this type of LAUP is a "walk-in, walk out" procedure that typically does not require any anaesthesia. In this paper, clinical experience is reported on the use of an Nd: YAG/Er: YAG dental laser system to perform the LAUP procedure to reduce symptoms of sleep-disordered breathing.
\end{abstract}

\section{Introduction}

Snoring and Sleep Disordered Breathing affects millions of Americans, both adults and children [1,2]. The signs and symptoms are the result of partial or complete collapse of the upper airway during sleep [3]. The structures involved in our protocol include the floor of the mouth, soft palate, uvula, and the base of the tongue [4]. The goal of the treatment is to decrease the amount of blockage of the upper airway [5]. Dentists are in a great position, as part of a team, to help screen and in many cases treat these problems with airway health management. Helping patients improve their sleep can profoundly improve their health, quality of life and the well-being of their loved ones. Both adults and children suffer from chronic snoring and other forms of sleep disordered breathing. A multitude of short- and longterm health issues can arise. The "Gold Standard" for the treatment of Sleep Apnea is the CPAP device, followed by Oral Appliance Therapy. We have found after many years of providing Dental Sleep Medicine, that many patients cannot attain the level of health that they need with just one treatment modality also compliance issues arise with both of these modalities as time goes on.

Obstructive sleep apnea is defined by the American Academy of Sleep Medicine as recurrent collapse of the upper airway during sleep, resulting in total (apnea) or partial (hypopnea) reduction in air flow. Changes in the mechanical physiology of respiration during sleep, including marked relaxation of upper airway muscles, result in doubling of the total upper airway resistance compared to wakefulness. The upper airway includes the portion of the respiratory system between the bars and proximal trachea. Multiple sites of potential obstructionist within the upper airway including the retropalatal and retroglossal area. Without DISE (Drug induced sleep endoscopy), it is very difficult to locate the exact location of airway collapse in SDB. This protocol takes this into consideration, by addressing most of the areas involved in concentric collapse. Dr. Friedman has discussed that his research shows that $75 \%$ of obstruction is caused by the tongue and $25 \%$ is palate etc [6]. Nightlase ${ }^{\text {Tix }}$ results in improved nasal breathing which has numerous benefits Comparing mouth breathing vs nasal breathing. Because of mouth breathing muscles and orofacial functions tend to adapt to a disordered breathing pattern. Nasal breathing filters out irritants, smooths air flow and enable improved blood /gas transfer in the lungs, but also help support optimal orofacial development. Patients who are not sleeping, breathing, and chewing well cannot be healthy. Retraining of the oral-facial muscles can help enable better sleep and breathing [7]. Tongue position is important also, genioglossus muscle lower fibres protrude the tongue, the geniohyoid muscle brings the hyoid bone superiorly and anteriorly this dilates the airway and aids in respiration. The digastric muscle elevates the hyoid bone. Addressing the floor of the mouth in the protocol helps with tongue position. Of the commercially available hard and soft tissue lasers, only the Nightwalker (Fotona, San Clemente, CA) combines two proven wavelengths, Nd:YAG and Er:YAG, wavelengths with unrivaled power and precise pulse control resulting in high levels of efficacy for a wide range of procedures. With this advanced level of performance comes significant patient comfort. The following procedure has been developed to take advantage of these attributes. The "Gold Standard" for the treatment

*Correspondence to: Harvey S. Shiffman, Boynton Laser Dental Center, Nova South-eastern College of Dental Medicine, 8200 Jog Road, Boynton Beach, Florida 33472, USA, E-mail: nocavat@aol.com

Key words: sleep-disordered breathing, SDB, snoring, laser-assisted uvulopalatoplasty, LAUP, nightlase, Nd: YAG, Er: YAG, nightlase C3

Received: January 08, 2020; Accepted: January 20, 2020; Published: January 27, 2020 
of sleep disordered breathing is the CPAP type device. Following that in 1981 was the introduction of Mandibular Advancement Devices (MAD). Compliance with both of these treatment modalities shows a reduction in compliance over time and significant side effects. However, the NIGHTLASE ${ }^{\text {ma }}$ C3 or Circumferential Snoring and Sleep Apnea Reduction Therapy protocol is a unique approach to treatment using the Fotona Lightwalker dental laser with a proprietary protocol and handpieces. Another positive benefit is the 24 hour a day improvement in nasal breathing that occurs Vs CPAP and MAD.

NIGHTLASE $^{\mathrm{ma}}$ uses the photothermal capabilities of the Lightwalker laser to convert and initiate the formation of new and more elastic collagen [8]. The target mucosal tissues are the oropharynx, soft palate, and uvula, back of the tongue and the floor of the mouth. The proprietary "Smooth Mode" pulse characteristics create a nonablative heat generation or "Heat Shock" that initiates the conversion of existing collagen to more elastic and organized forms and also initiates "neocollagenesis", the creation of new collagen in the fibroblast cells [8]. The effect of the laser energy as it penetrates (by transmission) deeper into the tissues is a low level photobiomodulation that directly affects the fibroblast cells and has been found to stimulate protein production from quiescent cells [9]. This process results in a visible elevation of the soft palate and uvula and tightening of the oropharyngeal tissues resulting in an improvement in the upper airway volume. Following the Nightlase protocol we are able to tighten up the facia and mucosa over the following muscles palatoglossus, palatopharyngeus, elevator veli palatini, tensor veli palatini. For the soft palate, palatoglossus and palatopharyngeus muscles, their fixed hard origins are at the hard palate. When they are tightened up with laser energy, they will move toward the hard palate. When the soft palate shrinks from the laser energy, it will shrink upward and forward to the hard palate which in turn will open up the nasopharyngeal airway and reduce snoring. The palatopharyngeus muscle, which is attached to the soft palate, also moves upward and forward with the contraction of the soft palate. Some believe that the palatopharyngeus muscle has a role in upper airway dilation. When we tighten up the palatoglossus muscle, the posterior part of tongue will elevate upward and slightly forward to the hard palate which in turn will improve the oropharyngeal airway, the posterior tongue posture and seal the mouth-to-pharynges air passage. As a result of all these actions, the nose-to-pharynges air passage will be enlarged, and the mouth-to-pharynges air passage will be reduced. Nightlase treatment helps patients to sleep better and breathe better through their noses. Nasal breathing has many documented health benefits related to daytime airway and sleep. However, if the nasal cavity and airways are small and inadequate due to an underdeveloped maxilla and premaxilla, then over time the body will lower the tongue posture to occupy the lower arch in order to reopen the mouth-topharynges air passage for better air supply. We feel the Nightlase treatment for these patients with restricted maxilla and nasal cavity will need more frequent touch up treatments. Jaw development treatments such as ALFs, DNA Appliances, and other functional appliances will work well with the Nightlase treatment. The jaw development with these functional appliances has been shown to help to increase the bony opening of the nasal cavity. There is an ALF article in the 2009 IAO (International Association of Orthodontics) journal documented the nasal cavity improvement on the PA cephalometric radiographs. Myofunctional exercises on correct swallowing are also essential to prevent long term relapse. In some cases, lingual frenectomies are also indicated to facilitate correct swallowing and correct tongue posture at rest and should always be evaluated as part of our clinical exam. The Nightlase protocol has many promising values will help patients with breathing issues and craniofacial pain also think that tightening up the elevator veli palatini and tensor veli palatini with laser energy through the soft palate helps to open up the Eustachian tube and reduces the pressure in the middle ear which may have an effect on some forms of tinnitus. Diaphragmatic nasal breathing is important, as is tongue position to help patients maintain their health, and myofunctional therapy is indicated for this as well. The effect of the laser energy as it penetrates (by transmission) deeper into the tissues is a low level photobiomodulation that directly affects the fibroblast cells and has been found to stimulate protein production from quiescent cells [9].

This process results in a visible elevation of the soft palate and uvula and tightening of the oropharyngeal tissues resulting in an improvement in the upper airway volume. Following the Nightlase protocol we are able to tighten up the facia and mucosa over the following muscles: palatoglossus, palatopharyngeus, elevator veli palatini, tensor veli palatini. For the soft palate, palatoglossus, and palatopharyngeus muscles, their fixed hard origins are at the hard palate. When they are tightened up with laser energy, they will move toward the hard palate. When the soft palate shrinks from the laser energy, it will shrink upward and forward to the hard palate which in turn will open up the nasopharyngeal airway and reduce snoring. The palatopharyngeus muscle, which is attached to the soft palate, also moves upward and forward with the contraction of the soft palate [10]. Some believe that the palatopharyngeus muscle has a role in upper airway dilation. When we tighten up the palatoglossus muscle, the posterior part of tongue will elevate upward and slightly forward to the hard palate which in turn will improve the oropharyngeal airway, the posterior tongue posture and seal the mouth-to-pharynges air passage. As a result of all these actions, the nose-to-pharynges air passage will be enlarged, and the mouth-to-pharynges air passage will be reduced. Nightlase treatment helps patients to sleep better and breathe better through their noses. Nasal breathing has many documented health benefits related to daytime airway and sleep [11]. However, if the nasal cavity and airways are small and inadequate due to an underdeveloped maxilla and premaxilla, then over time the body will lower the tongue posture to occupy the lower arch in order to reopen the mouth-topharynges air passage for better air supply. We feel the Nightlase treatment for these patients with restricted maxilla and nasal cavity will need more frequent touch up treatments. Jaw development treatments such as ALFs, DNA Appliances, and other functional appliances. will work well with the Nightlase treatment. The jaw development with these functional appliances has been shown to help to increase the bony opening of the nasal cavity. There is an ALF article in the 2009 IAO (International Association of Orthodontics) journal documented the nasal cavity improvement on the PA cephalometric radiographs. Myofunctional exercises on correct swallowing are also essential to prevent long term relapse [12]. In some cases, lingual frenectomies are also indicated to facilitate correct swallowing and correct tongue posture at rest and should always be evaluated as part of our clinical exam. The Nightlase protocol has many promising values. It will help patients with breathing issues and craniofacial pain. It is our belief, that tightening up the elevator veli palatini and tensor veli palatini with laser energy through the soft palate helps to open up the Eustachian tube and reduces the pressure in the middle ear which may have an effect on some forms of Tinnitus [13]. Diaphragmatic nasal breathing is very important, as is tongue position to help patients maintain their health, and myofunctional therapy is indicated for this as well.

NIGHTLASE $^{\mathrm{m}}$ therapy is indicated for cases when the patient has been diagnosed with chronic snoring, UARS or mild to moderate sleep apnea and either cannot or chooses not to wear a MAD appliance or 


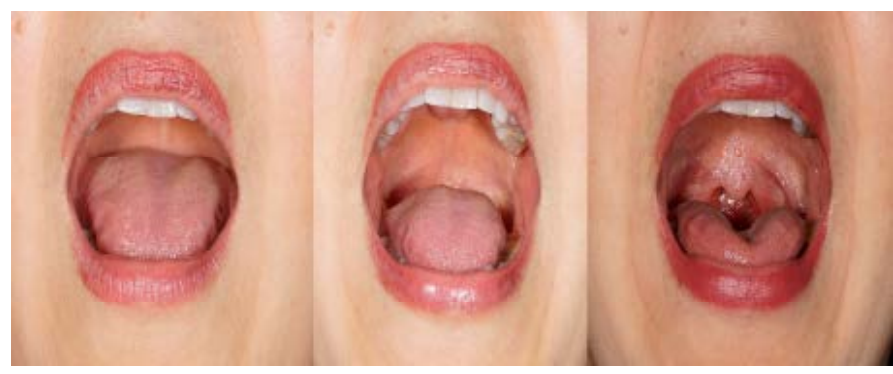

Figure 1. Astrid b4 after and 14 hrs PO NL

CPAP device. It can also be used in co-therapy with those devices and represents a less invasive alternative to current surgical, chemical or radiosurgical options that may require hospitalization, general anesthesia or soft tissue removal. NIGHTLASE ${ }^{\text {ta }}$ has a significant success rate in producing a positive change in sleep patterns. Research published by Miracki and Visintin [7] has shown that it can reduce and attenuate snoring and provides an effective non-invasive modality to lessen the effects of Obstructive Sleep Apnea. As with any treatment, there are potential risks with laser treatment. However, the risks are minimal and certainly less then alternative therapies if the protocol is followed correctly. NIGHTLASE ${ }^{m}$ therapy is not a permanent alteration and lasts anywhere from 6-12 months and is easily touched up at follow up appointments (Figure 1).

In 2013 we completed a pilot study that addressed snoring only with 12 patients. Twelve months follow up showed $30-90 \%$ reduction in snoring tone and volume. The lower percentages were smokers, obese patients, and those with severe OSA. Follow-up studies with polysonography using HST are in process as are pharyngometer studies. Both of which have shown significant positive changes in the early results [14].

A recently published pilot research study by Lee and Lee [9] has shown through colorized 3D CT imaging, the volumetric positive changes after Nightlase ${ }^{\text {tw }}$ treatment. This has helped support the clinical results, and the authors have follow-up studies with 3D CT, polysonography and a larger group of patients in process. We consider Nightlase to be another tool in our treatment "toolbox" giving us more options and the possibility of better results when used in combination with MAD's and CPAP for those patients that may not get adequate improvements from those therapies alone. Nightlase is also an interim therapy for children, adolescents, and teenagers while they undergo craniofacial development to enlarge their airways. We are excited to present these modern, minimally invasive, and more natural treatment modalities to the dental community. Using the Lightwalker laser, we can now offer our patients health improvements that reach beyond restorative and rehabilitative dentistry [15].

\section{Financial disclosure}

The author has no financial interest in the products mentioned in this article.

\section{References}

1. A report of the National Commission on Sleep Disorders Research (1995) Wake Up America: A National Sleep Alert. Washington, D.C. U. S. Government Printing Office.

2. Young T, Peppard PE, Gottlieb DJ (2002) Epidemiology of obstructive sleep apnea: a population health perspective. Am J Respir Crit Care Med 165: 1217-1239. [Crossref]

3. Lattimore JD, Celermajer DS, Wilcox I (2003) Obstructive sleep apnea and cardiovascular disease. J Am Coll Cardiol 41:1429-1437. [Crossref]

4. Courey MS, Fomin D, Smith T, Huang S, Sanders D, et al. (1999) Histologic and physiologic effects of electrocautery, $\mathrm{CO} 2$ laser, and radiofrequency injury in the porcine soft palate. Laryngoscope 109: 1316-1319. [Crossref]

5. Fomin D, Nicola E, Oliver C, Farci M, Dibbern R, et al. (2007) Collagen type analysis in the soft palate after surgical intervention with $\mathrm{CO}(2)$ laser and radiofrequency ablation. Photomed Laser Surg 25: 449-454. [Crossref]

6. Valcheva Z, Arnautska H, Dimova M, Ivanov G, Atanasova I (2018) The role of Mouth Breathing on dentition development and formation. J IMAB 24: 1878-1882.

7. Liu H, Dang Y, Wang Z, Chai X, Ren Q (2008) Laser induced collagen remodeling: a comparative study in vivo on mouse model. Lasers Surg Med 40: 13-19. [Crossref]

8. Vanderveken OM (2013) Drug-induced sleep endoscopy (DISE) for non-CPAP treatment selection in patients with sleep-disordered breathing. Sleep Breath 17: 13-14. [Crossref]

9. Miracki K, Vizintin Z (2013) Nonsurgical minimally invasive Er: YAG laser snoring treatment. $J$ Laser and Health Academy 1: 36-41.

10. Cameron Y. S. Lee, Cameron C. Y. Lee (2015) Evaluation of a non-ablative Er: YAG laser procedure to increase the oropharyngeal airway volume: A pilot study. Dent Oral Craniofac Res 1: 56-59.

11. Spock C, Metelitsa AI, Kaufman J, Green JB (2012) Lasers and Lightsources to Activate Fibroblasts, Cosmet Dermatol 25: 27-33.

12. Recinto C, Efthemeou T, Boffelli PT, Navalta JW (2017) Effects of Nasal or Oral Breathing on Anaerobic Power Output and Metabolic Responses. Int J Exerc Sci 10: 506-514. [Crossref]

13. Delz E (2009) The ALF (Advanced Lightwire Functional Appliance) creating facial beauty and balance. Int J Orthod Milwaukee 20: 23-27. [Crossref]

14. John Mersch (2018) Eustachian Tube Dysfunction.

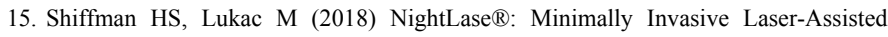
Uvulopalatoplasty .J Laser and Health Academy 1: 39-44.

Copyright: (C2020 Shiffman HS. This is an open-access article distributed under the terms of the Creative Commons Attribution License, which permits unrestricted use, distribution, and reproduction in any medium, provided the original author and source are credited. 